\title{
AVALIAÇÃO DO IMPACTO DE EVENTOS CLIMÁTICOS EXTREMOS NOS OCEANOS PACÍFICO E ATLÂNTICO SOBRE A ESTAÇÃO CHUVOSA NO NORDESTE DO BRASIL
}

\author{
DAISY BESERRA LUCENA ${ }^{1}$; MANOEL F. GOMES FILHO ${ }^{2}$; JACQUES SERVAIN ${ }^{3}$ \\ ${ }^{1}$ Centro de Desenvolvimento Sustentável do Semiárido da Universidade Federal de Campina Grande \\ (UFCG), Sumé, PB, Brasil. \\ ${ }^{2}$ Departamento de Ciências Atmosféricas da UFCG, Campina Grande, PB, Brasil. \\ ${ }^{3}$ Institut de Recherche pour le Développement (IRD-UR182), França, Cientista Visitante na Fundação \\ Cearense de Meteorologia e Recursos Hídricos (FUNCEME), Fortaleza, CE, Brasil. \\ daisylucena@yahoo.com.br,mano@dca.ufcg.edu.br, jacques.servain@gmail.com
}

Recebido Maio 2008 - Aceito Janeiro 2011

\begin{abstract}
RESUMO
O impacto dos eventos climáticos extremos na região dos Oceanos Pacífico (El Nino - EN; La Nina - LN) e Atlântico (Gradiente meridional - GRADM) sobre a precipitação de três sub-regiões distintas do Nordeste do Brasil (NEB), é avaliado durante o período 1948 a 1997. A análise baseou-se em dados de precipitação observada e simulada pelo Modelo de Circulação Geral da Atmosfera ARPEGE. Os resultados mostram que a precipitação sobre as três sub-regiões apresenta uma grande variabilidade independente dos episódios EN ou LN, com uma predominância de anos secos (chuvosos) nas subregiões leste e norte do NEB antes (depois) de 1976, isto é, quando os episódios de LN (EN) foram mais numerosos. A resposta interanual da precipitação é mais forte quando o GRADM é positivo (induzindo secas) ou negativo (períodos chuvosos) sobre a região NEB, demonstrando uma forte influência climática do Atlântico versus o Pacífico. Combinando as influências simuladas no Pacífico e Atlântico, bem como os dados da precipitação observada no período verificou-se que, durante os anos de EN o oceano tropical é responsável pela variabilidade das chuvas sobre o NEB (especialmente as sub-regiões Norte e Leste). Por outro lado, durante episódios de LN o Oceano Atlântico tropical é a principal contribuição e a influência do Oceano Pacífico é relativamente modesta.
\end{abstract}

Palavras-Chaves: Precipitação, Nordeste do Brasil, MCGA, Eventos Climáticos.

\begin{abstract}
IMPACT EVALUATION OF THE EXTREME CLIMATIC EVENTS IN THE PACIFIC AND ATLANTIC OCEANS ON THE NORTHEAST BRAZIL RAINY SEASON

Impact of extreme climatic events in the Pacific (El Niño, EN; La Niña, LN) and Atlantic (Meridional SST Gradient, GRADM) on the rainfall of three sub-regions over the Northeast Brazil - NEB (Northern NEB, Eastern NEB and Centre/Southern NEB) are evaluated during the period 1948 to 1997. The analysis includes observed precipitation data and simulated rainfall outputs derived from the ARPEGE Atmospheric General Circulation Model (AGCM). The results show that the precipitation over the three sub-regions presents a high variability independent of LN or EN episodes, with a predominance of dry (wet) years in the sub-regions Eastern and Northern of NEB before (after) 1976, i.e. when LN (EL) episodes were more numerous. The interannual rainfall response is stronger when GRADM is positive (inducing dry NEB) or negative (inducing wet NEB), thus showing a stronger climatic influence of the Atlantic vs. the Pacific. Combining simulated influences of Pacific and Atlantic as well as observed precipitation data over the entire period, it was found that during EN years the global tropical ocean is responsible for the rainfall variability over the NEB (especially in the Northern and Eastern sub-regions). On the other hand, during LN years, the Atlantic tropical Ocean is the main contribution, and the tropical Pacific Ocean influence is relatively modest.
\end{abstract}

KEYwords: Rainfall, Northeast Brazil, AGCM, Extreme climatic events. 


\section{INTRODUÇÃO}

A análise do comportamento das chuvas na Região Nordeste do Brasil (NEB) se reveste de enorme importância, devido à grande irregularidade apresentada em escala de tempo e espaço, não somente sob o enfoque climático, mas também, pelas conseqüências de ordem econômica, social e cultural advindas.

A variabilidade do clima sobre o NEB é modulada por padrões atmosféricos e oceânicos de grande escala que se processam (conjuntamente ou não) sobre os Oceanos Pacífico e Atlântico Tropicais. O exemplo clássico de interação atmosfera/ oceano é o fenômeno El Niño/Oscilação do Sul (ENOS). O El Niño (EN) é caracterizado pelo aquecimento anômalo da camada superficial e sub-superficial do Oceano Pacífico Central e Leste (Rasmusson e Carpenter, 1982). Condição oposta caracteriza os eventos de La Niña (LN). A oscilação do Sul (OS) é uma variação anômala da pressão atmosférica tropical, sendo uma resposta aérea do EN, associada à mudança na circulação geral da atmosfera (Aragão, 2004). Essa interação tem sido considerada uma das causas de variações climáticas sobre o NEB (Rao e Hada, 1990; Aceituno, 1988).

Esta conexão do EN com o NEB ocorre através da circulação atmosférica, tal que a seca relacionada ao evento é atribuída a um deslocamento, para leste, da circulação de Walker, com movimentos ascendentes anômalos (aumento da convecção) sobre o Pacífico equatorial central e leste, e movimentos descendentes (inibição da convecção) sobre o Atlântico Tropical (AT) e a área continental do NEB (Hastenrath, 1976; Kousky et al., 1984; Kayano at al., 1988; Ropelewski e Halpert, 1987 E 1989; Kiladis e Diaz, 1989; Rao e Hada, 1990). Padrões anômalos contrários na circulação atmosférica, Temperatura da Superfície do Mar (TSM) e precipitação no NEB são observados em episódios de LN (Kousky e Ropelewski, 1989; Kayano e Andreoli, 2004).

Além da influência do oceano Pacífico, a variabilidade da TSM no Oceano Atlântico Tropical também apresenta sua importância e impacto sobre o clima da região NEB (Markham e Mclain, 1977; Hastenrath e Heller, 1977; Moura e Shukla, 1981; Andreoli e Kayano, 2004). O Atlântico é controlado por um gradiente da TSM meridional ou inter-hemisférico (GRADM), conhecido também como padrão de dipolo (Moura e Shukla, 1981; Servain, 1991). Essa variabilidade ocorre em escala interanual e decadal, e está ligada à variação meridional da Zona de Convergência Intertropical (ZCIT), que é um dos principais mecanismos que provoca precipitação no norte $\mathrm{e}$ também sobre o leste do NEB.

$\mathrm{Na}$ ausência dos eventos sobre o Pacífico (EN ou LN), a precipitação no NEB se correlaciona mais fortemente com as anomalias da TSM (ATSM) do Atlântico Tropical Sul - ATS do que com as do Atlântico Tropical Norte - ATN (Saravanan e Chang, 2000). Alguns estudos como os de Hastenrath e Heller (1977), Hastenrath (1978) e Moura e Shukla (1981), mostram que em anos de secas, as ATSM no Atlântico Tropical mostram um comportamento de GRADM positivo (ATSM positivas ao norte e negativas ao sul do equador), para anos mais chuvosos esse comportamento é invertido, isto é, GRADM negativo. Para anos concordantes sobre o Pacifico e Atlântico, ou seja, EN e GRADM positivo ou LN e GRADM negativo, a relação entre as ATSM do Atlântico Tropical e a precipitação do NEB é mais forte e as amplitudes das anomalias de precipitação são maiores e mais significativas, sendo observadas condições de seca e chuva, respectivamente, em torno ou maiores do que a média. Em anos discordantes (EN e GRADM negativo ou LN e GRADM positivo) esta relação é mais fraca, pois o Atlântico Tropical age no sentido contrário ao evento no Pacífico, limitando ou até mesmo invertendo o sinal das anomalias de precipitação sobre o NEB (Pezzi e Cavalcanti, 2001, Giannini et al., 2004).

Diante do exposto, esse trabalho tem como objetivo avaliar as condições das precipitações sobre três sub-regiões com diferentes regimes de chuvas sobre a região NEB em anos de eventos climáticos sobre os Oceanos Pacífico e Atlântico. Esse objetivo será realizado através da análise sazonal das TSM na região do Niño 3.4 e das TSM no ATN e ATS, além da precipitação observada e simulada por um Modelo de Circulação Geral da Atmosfera (MCGA) ARPEGE (Déqué et al. 1994). Será comparado também, o comportamento (observado e simulado) da resposta da precipitação durante 4 cenários: anos de EL Niño, La Niña, Gradiente meridional positivo e Gradiente meridional negativo.

\section{DADOS E METODOLOGIA}

A região em estudo é o Nordeste do Brasil (NEB), que está situada na zona tropical, entre os meridianos de $35^{\circ}$ e $47^{\circ} \mathrm{We}$ os paralelos de $1^{\circ}$ e $18^{\circ} \mathrm{S}$. Marques et al. (1983) propuseram que a distribuição espacial da precipitação nessa região é dividida em três regimes básicos (Figura 1a), dessa forma, neste trabalho o NEB foi subdividido em retângulos que tentam reproduzir essas sub-regiões conforme visualizado na Figura 1b. Tendo em vista diversos estudos como, por exemplo, Pezzi e Cavalcanti (2001), Giannini et al. (2004), que mostram o impacto maior dos eventos ENSO e GRADM sobre o norte e leste do NEB (sub-regiões 1 e 2), divide-se com mais precisão essas subregiões, apesar da utilização de retângulos que torna a divisão geral, e para não multiplicar a quantidade das sub-regiões, a sub-região 3 abrange o "restante" do NEB, pegando o norte de Minas Gerais, contudo isso não afetará, em princípio, a análise. $\mathrm{O}$ intuito é o de observar maiores detalhes da variabilidade 
climática e a influência dos fenômenos em escalas locais e remotas, já que nessas sub-regiões o período chuvoso, foco do nosso estudo, ocorre em períodos distintos, sugerindo que mais de um mecanismo de circulação geral seja responsável por essas precipitações.

As sub-regiões são definidas, praticamente, da seguinte maneira:

- Sub-região 1 (entre $1,5^{\circ} \mathrm{S}$ e $8^{\circ} \mathrm{S}$ e $37^{\circ} \mathrm{W}$ e $46,2^{\circ} \mathrm{W}$ ) abrange o norte do NEB, a principal estação chuvosa ocorre de fevereiro a maio - FMAM (Molion e Bernardo, 2002). Tem a ZCIT como o principal mecanismo produtor de chuvas, durante os meses de março e abril (Uvo, 1989; Nobre e Shukla, 1996; Molion e Bernardo, 2002), além das Perturbações Ondulatórias dos Alísios - POAs e convecção local. A sub-região é caracterizada por um regime pluviométrico que varia de 400 $\mathrm{mm} /$ ano no inferior, a mais de $2.000 \mathrm{~mm} / \mathrm{ano}$ no litoral (Nobre e Molion, 1988);

- Sub-região 2 (entre $5^{\circ} \mathrm{S}$ e $11^{\circ} \mathrm{S}$ e $34,5^{\circ} \mathrm{W}$ e $37^{\circ} \mathrm{W}$ ) leste do NEB, o seu período mais chuvoso está compreendido entre os meses de abril a julho (AMJJ), e está relacionado à maior atividade de circulação de brisa que advecta bandas de nebulosidade para o continente, possuindo um total pluviométrico variando de 600 a $3.000 \mathrm{~mm} /$ ano (Nobre e Molion, 1988). Observa-se também a influencia das ondas de leste ou distúrbios ondulatórios de leste que são responsáveis pelas chuvas entre os meses de maio a agosto (Yamazaki e Rao, 1977); e

- Sub-região 3 (entre $8^{\circ} \mathrm{S}$ e $18,4^{\circ} \mathrm{S}$ e $37^{\circ} \mathrm{S}$ e $46,2^{\circ} \mathrm{W}$ ) região centro/sul do NEB, é bom lembrar que na divisão essa sub-região inclui também uma parte do semi-árido no norte de Minas Gerais. Tem entre novembro a fevereiro (NDJF) o período mais chuvoso (Molion e Bernardo, 2002). O principal mecanismo de precipitação é a estacionariedade dos sistemas frontais alimentados pela umidade proveniente do ATS, que


Figura 1 - (a) Regime de precipitação sobre o Nordeste do Brasil com seus meses mais chuvosos: (12) Dezembro, (3) Março e (5) Maio. Fonte: Adaptada de Marques et al. (1983). (b) Subdivisões da região NEB: sub-região 1 (norte do NEB); sub-região 2 (leste do NEB); e sub-região 3 (centro-sul do NEB). definem a Zona de Convergência do Atlântico Sul - ZCAS, sistemas pré-frontais, convecção local e brisas mar e terra (Molion e Bernardo, 2002). Possui um regime pluviométrico que varia de $600 \mathrm{~mm} /$ ano no interior, a mais de $3.000 \mathrm{~mm} / \mathrm{ano}$ no litoral (Nobre e Molion, 1988).

Ademais, existem outros sistemas que organizam a convecção em escala sinótica que atuam na região NEB, tais como os vórtices ciclônicos de altos níveis (VCAN) que provocam precipitação durante a primavera, verão e outono (setembro a abril), com ocorrência máxima no mês de janeiro (Kousky e Gan, 1981) e a oscilação 30-60 dias ou oscilação de Madden-Julian (OMJ) (Kousky e Kayano, 1994). Na mesoescala tem-se os complexos convectivos de mesoescala (CCM) e as brisas marítima e terrestre que influenciam todas as subregiões, além das circulações orográficas e pequenas células convectivas que constituem os fenômenos de microescala (Molion e Bernardo, 2002).

Utilizaram-se dados de precipitação mensais conhecidos como reconstrução da precipitação sobre os continentes (PRECL), distribuídos numa grade de $2.5^{\circ}$ de latitude e longitude para o período de 1948 a 2000, e são derivados de dados observados coletados em 17.000 pluviômetros, fornecidos pela Rede Global Histórica Climatológica $-2^{\mathrm{a}}$ versão $(\mathrm{GHCN} 2)^{1}$ e pelo Sistema de Monitoramento de Anomalias Climáticas $(\mathrm{CAMS})^{2}$. Esses dados são disponibilizados pelo Climate Prediction Center (CPC) da NOAA ${ }^{3}$, obtidos através do sitio $<$ http://iridl.ldeo.columbia.edu/ $>$. Posteriormente, esses dados serão referenciados por CPC. Maiores detalhes sobre esses ver Chen et al. (2002).

Do mesmo modo, foram utilizados dados de precipitação gerados por simulações do modelo de circulação geral ARPEGE ${ }^{4}$-Climat, desenvolvido conjuntamente por MétéoFrance e o Centro Europeu de Previsão do Tempo (ECMWF) ${ }^{5}$. Essas são forçadas por diferentes configurações da TSM para o período de 1948-1997, definidos numa grade de $2.8^{\circ}$ x $2.8^{\circ}$. Dequé et al. (1994) descreveram as características gerais desse modelo. Serão utilizados dois tipos de experimentos, o experimento GOGA (Global Ocean - Global Atmosphere) que é uma média do conjunto de 8 simulações atmosféricas, forçado por TSM globais observadas e extensão de gelo-mar e o experimento AOGA (Atlantic Ocean-Global Atmosphere) que é uma média do conjunto de 4 simulações atmosféricas executadas com as mesmas TSM, exceto que a forçante é especificada apenas sobre o Atlântico ao norte de $45^{\circ} \mathrm{S}$, ou seja, só com o campo da TSM observada sobre o Atlântico e a climatologia nos outros oceanos. É importante ressaltar que esses dados foram gerados por Cassou (2001), com o intuito de

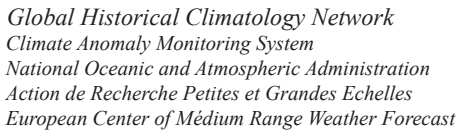


verificar a variabilidade atmosférica de baixa freqüência sobre a região norte do Atlântico - Europa.

A região da TSM sobre o Pacífico equatorial considerada como representativa da intensidade dos eventos ENOS será a Niño 3.4, por possuir temperaturas médias superiores às da região Niño 3, estar próxima da piscina quente e principais centros de convecção do Pacífico equatorial. Além do mais, é o índice mais difundido operacionalmente para fins de monitoramento e previsão dos eventos ENOS e suas teleconexões (Trenberth, 1997). Da mesma forma, segundo Hanley et al. (2003), os índices de Oscilação do Sul, Niño 3.4 e Niño 4 são igualmente sensíveis aos eventos de EN e melhores para caracterizá-los do que outros.

Os anos de EN e $\mathrm{LN}$ foram selecionados tendo com base a lista do CPC/NOAA, e se baseiam num limiar de $+/-0.5^{\circ} \mathrm{C}$ para o Índice Nino, para o período de 1950-2007. Foram selecionados 14 anos de $\mathrm{EN}(57 / 58,63 / 64,65 / 66,68 / 69,69 / 70,72 / 73,76 / 77$, $77 / 78,82 / 83,86 / 87,87 / 88,90 / 91,91 / 92$ e $94 / 95)$ e 13 anos de LN (49/50, 50/51, 54/55, 55/56, 64/65, 70/71, 71/72, 73/74, $74 / 75,75 / 76,84 / 85,88 / 89$ e 95/96). Os anos selecionados também estão em coerência com os de outras fontes, como por exemplo, CPTEC/INPE (http://www.cptec.inpe.br/enos).

Por outro lado, os anos correspondentes aos anos com GRADM positivo ou negativo foram escolhidos com base no cálculo do índice proposto por Servain (1991), que consiste na diferença entre as médias da TSM na área delimitada na bacia norte do Atlântico por $60^{\circ} \mathrm{W}-20^{\circ} \mathrm{W}, 5^{\circ} \mathrm{N}-28^{\circ} \mathrm{N}$, menos a área na bacia sul $35^{\circ} \mathrm{W}-5^{\circ} \mathrm{E}, 20^{\circ} \mathrm{S}-5^{\circ} \mathrm{N}$. A demarcação da linha de separação das duas bacias é definida em $5^{\circ} \mathrm{N}$, pois essa linha oferece uma boa representação do equador térmico (Servain, 1991). Os dados para realização desse cálculo foram os dados do GISST (dados de entrada do modelo ARPEGE). Foram selecionados como anos de ocorrência do GRADM positivo aqueles em que o índice, acima descrito, apresentou valor acima de $0,5^{\circ} \mathrm{C}$ e GRADM negativo os valores abaixo de $-0,5^{\circ} \mathrm{C}$, durante pelo menos dois meses consecutivos entre os meses de janeiro a junho. Dessa forma, foram obtidos 10 episódios com GRADM positivo (50/51, 55/56, 57/58, 65/66, 69/70, 77/78, 79/80, 80/81, 82/83 e 96/97) e 09 com GRADM negativo (70/71, 72/73, 73/74, 74/75, 83/84, 84/85, 85/86, 88/89 e 93/94).

\section{RESULTADOS E DISCUSSÕES}

\subsection{Anos de Eventos EI Niño}

A Figura 2 mostra o índice sazonal da TSM na região Niño 3.4 para os 14 episódios de El Niño selecionados, podendo-se observar a evolução e intensidade sazonal dos eventos nos trimestres SON, DJF, MAM e JJA. Nota-se que cada evento EN tem características próprias, não apresenta um mesmo mês de início e de fim, o que se pode observar, são anomalias da TSM positivas, pelo menos, nos dois primeiros trimestres ( $\mathrm{SON}$ e DJF), que correspondem à fase de desenvolvimento e madura

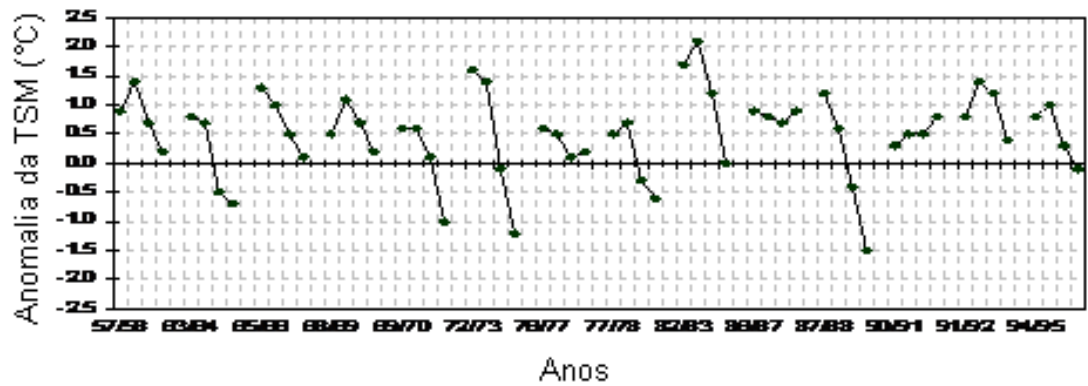

Figura 2 - Anomalia sazonal da TSM sobre a Região Niño 3.4 para 14 anos de evento El Niño. Os trimestres são, respectivamente, SON, DJF, MAM e JJA para cada ano.NEB: sub-região 1 (norte do NEB); sub-região 2 (leste do NEB); e sub-região 3 (centro-sul do NEB).

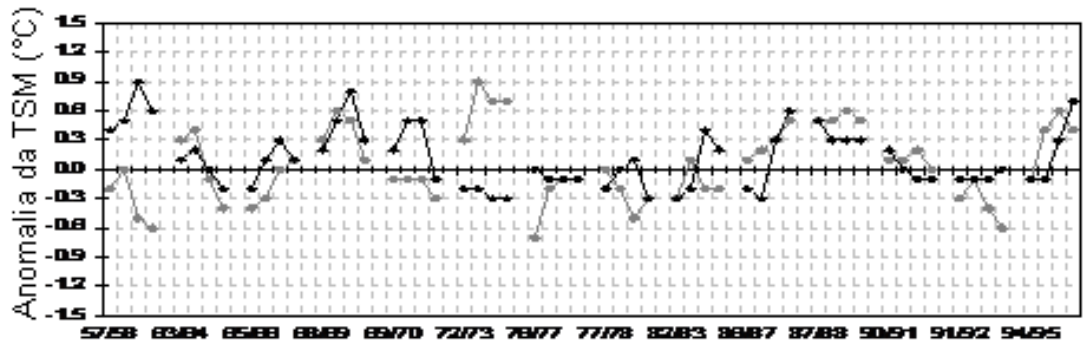

Anns

Figura 3 - Anomalia sazonal da TSM sobre o ATS (linha cinza) e o ATN (linha preta) para 14 anos de evento El Niño. Os trimestres são, respectivamente, SON, DJF, MAM e JJA para cada ano. 
do EN tal como descrito na literatura (Rasmusson e Carpenter, 1982; Wang, 2002).

Os índices sazonais para o Atlântico Norte (ATN) e Atlântico Sul (ATS) nos anos de EN podem ser observados na Figura 3. Percebe-se que o ATN apresenta, em geral, um sinal de anomalias positivas, o que está de acordo com outros estudos que revelam que o ATN sofre influência do EN (Penland e Matrosova, 1998; Sutton et al., 2000; Giannini et al., 2001; Andreoli, 2002; Mélice e Servain, 2003). A exceção pode ser visualizada nos EN ocorridos na década de 70 e no início da década de 90, quando as TSM sobre a bacia norte, estão ligeiramente abaixo da média. O ATS possui oscilações entre ATSM positivas e negativas. O GRADM mostra-se bem caracterizado nos anos 57/58, 69/70, $72 / 73,77 / 78$, porém também pode ser visualizado nos anos $65 / 66$ e 82/83. Após 1976, tem aumentado a freqüência de GRADM negativo com ocorrência de EN.

As anomalias sazonais das precipitações observadas do CPC em anos de EN para cada sub-região estão exibidas na



Figura 4 - Anomalia sazonal da precipitação para as três sub-regiões sobre o NEB com os dados observados do CPC/NOAA para anos de evento El Niño. (a) Sub-região 1 - Norte; (b) Sub-região 2 - Leste e (c) Sub-região 3 - Centro/Sul. Os trimestres são, respectivamente, SON, DJF, MAM e JJA para cada ano.

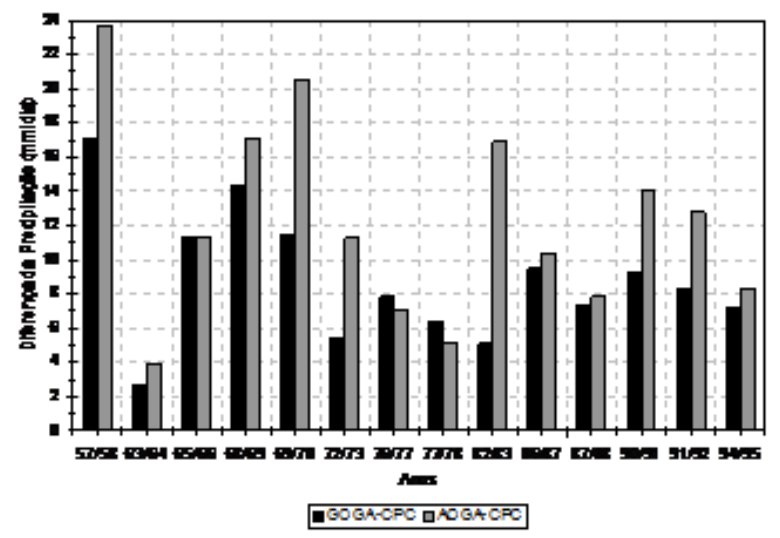

Figura 5 - - Diferença da precipitação (FMAM) entre as simulações e os dados observados do CPC (GOGA-CPC e AOGA-CPC) nos anos de El Niño para a sub-região 1 . 
Figura 4. Ela mostra que o período chuvoso de cada sub-região apresenta uma alta variabilidade da precipitação nesses anos de eventos EN. Para a sub-região 1 - norte (Figura 4a), no trimestre MAM, observa-se que existe um predomínio de precipitações abaixo da normal, o que está em concordância com estudos anteriores que revelam que essa sub-região é bastante afetada pelo fenômeno EN. Porém, os anos de 63/64, 72/73, 87/88, 94/95 apresentaram anomalias de precipitação positivas. Vale ressaltar que esses eventos EN foram de curta duração, e se observar, o GRADM estava negativo. Dessa maneira, pode ser verificada a influência entre os Oceanos Pacífico e Atlântico.

Para o leste do NEB (Figura 4b), foram analisados os trimestres MAM e JJA, através dos quais se verificam facilmente as fases relacionadas às mudanças ocorridas na década de 70 , na qual se observa que os períodos chuvosos, antes de 1978, apresentaram precipitações acima da média e depois valores, geralmente, abaixo da média climatológica.

Sobre a sub-região 3 - Centro/Sul (Figura 4c), observando o trimestre DJF (período chuvoso) em anos de $\mathrm{EN}$, as anomalias de precipitação, em geral, apresentaram-se pequenas e bem próximas da média. Apenas os anos 63/64, 69/70, 77/78 e 91/92 mostram anomalias positivas com maior intensidade e o ano de 86/87 com anomalias negativas. O que chama a atenção é o cruzamento das anomalias pelo eixo $x$, ou seja, a mudança de anomalias positivas para negativas, ou vice-versa, isso analisando os trimestres que compõem a pré e a estação chuvosa (SON e DJF, respectivamente). Uma explicação plausível pode ser devido à área delimitada que apresenta mais de um regime de precipitação.

A partir dos resultados das simulações GOGA e AOGA pode-se visualizar a contribuição de todos os oceanos (através da GOGA), bem como a atuação do Atlântico na simulação AOGA, já que, nessa simulação, foram excluídos os eventos extremos sobre o Pacífico. Sendo assim, através da diferença entre a precipitação simulada pelos dois experimentos e a precipitação observada, analisou-se qual o oceano apresenta uma maior contribuição para o melhor resultado da precipitação nos anos de eventos sobre os oceanos tropicais.

Durante os 14 anos de eventos EN, a diferença GOGACPC apresentou em 79\% dos eventos, a menor diferença em relação à AOGA-CPC (Figura 5). Observa-se que as maiores diferenças entre essas precipitações são encontradas nos anos de EN fortes (57/58, 72/73, 82/83, 90/91 e 91/92) o que era esperado, já que na simulação teste AOGA os eventos extremos são suprimidos. Desse modo, pode-se concluir que eventos EN com intensidade forte predominaram, contudo é bom sempre lembrar que, mesmo assim, outros fatores podem colaborar no impacto da precipitação na sub-região 1, como por exemplo, as condições da TSM sobre o oceano Atlântico. Nos anos de eventos moderados e fracos, fica bem claro que as diferenças tornam-se mais próximas, porém ainda pode ser verificado que a diferença GOGA-CPC apresenta os menores valores. Apesar da diferença entre GOGA-CPC e AOGA-CPC ser pequena, pode-se verificar a contribuição do AT em anos de EN, como por exemplo, nos anos de 1977 e 1978.

A Figura 6 mostra as diferenças da precipitação entre as simulações e a observada para a sub-região 2. Observar-se nessa figura que a simulação conseguiu reproduzir de forma razoável essa variável, em escala interanual nos anos de EN. Ao contrário do que mostra a Figura 5 para a sub-região 1, as maiores diferenças entre as simulações não foram só encontradas nos anos de EN considerados fortes, alguns EN de intensidade moderada também apresentaram diferenças intensas. O ano de 1973 chama a atenção por ser um ano de $\mathrm{EN}$ forte, em que as diferenças entre a precipitação observada e a simulada para as sub-regiões 1 e 2 são praticamente iguais, porém como observado na Figura 2, este evento apresentou uma desintensificação muito intensa, desde SON até JJA, e como a estação chuvosa dessa sub-região 2 ocorre na fase final do evento, isto pode ser uma explicação para este fato. Entretanto, em termos gerais, a diferença GOGA-CPC foi menor.

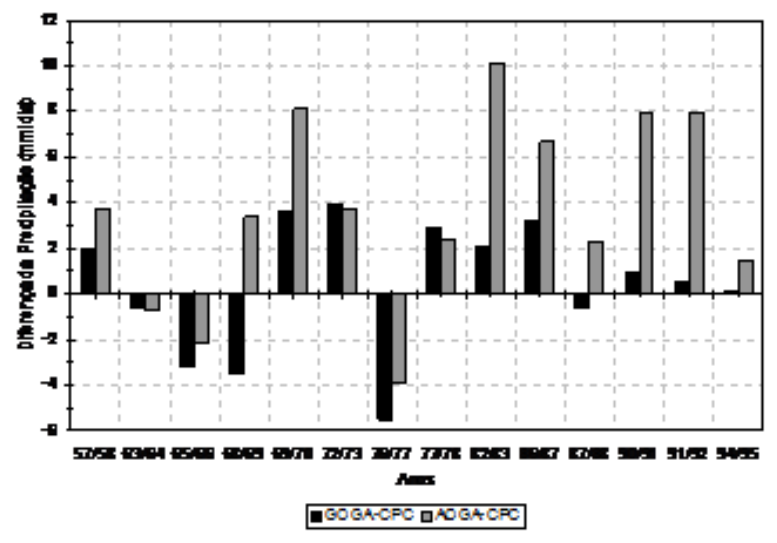

Figura 6 - Diferença da precipitação (AMJJ) entre as simulações e os dados observados do CPC (GOGA-CPC e AOGA-CPC) nos anos de El Niño para a sub-região 2 . 
Analisando a Figura 7, observa-se que, em comparação com as sub-regiões 1 e 2, a sub-região 3 apresentou as maiores diferenças. Nota-se, também, que os valores das diferenças entre as duas simulações (AOGA e GOGA) e a CPC são bem próximas, podendo ser um indicativo de que a principal fonte de umidade, para essa sub-região, seja o Oceano Atlântico.

\subsection{Anos de Eventos La Niña}

Da mesma forma que os eventos de EN, o fenômeno LN também tem suas características próprias para cada evento (Figura 8). Podem-se observar as anomalias negativas da TSM relacionadas ao resfriamento das águas do Pacífico Central Equatorial. Este evento já se encontra configurado no trimestre SON.

Observa-se que as anomalias da TSM no ATS (Figura 9) apresentaram-se, até o ano de 1976, predominantemente negativas, com exceção apenas do ano de 1973/74. Após 1976, nota-se uma mudança para anomalias positivas no ATS. Sobre o ATN, as anomalias oscilaram entre positivas e negativas durante o período 1949-1956. Entre 1965-1985 as anomalias foram predominantemente negativas nas duas bacias, ou seja, LN associadas a anomalias negativas. O GRADM é claramente definido em alguns anos do evento La Niña, tais como 55/56,

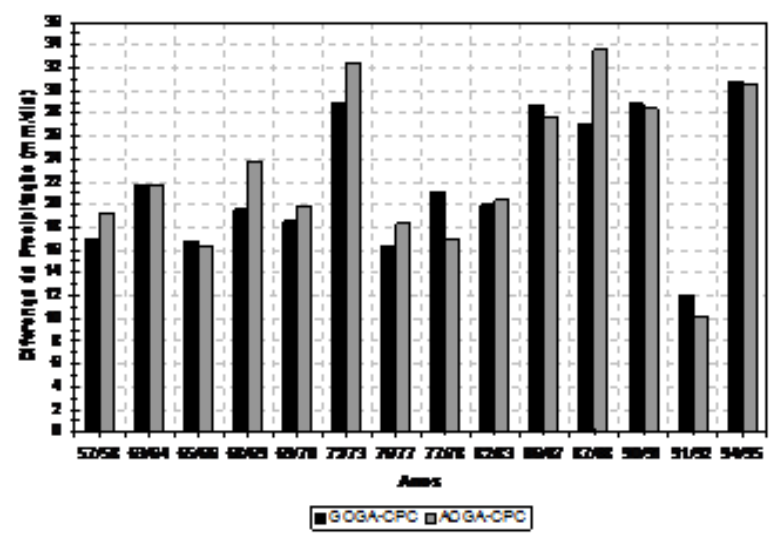

Figura 7 - Diferença da precipitação (NDJF) entre as simulações e os dados observados do CPC (GOGA-CPC e AOGA-CPC) nos anos de El Niño para a sub-região 3 .

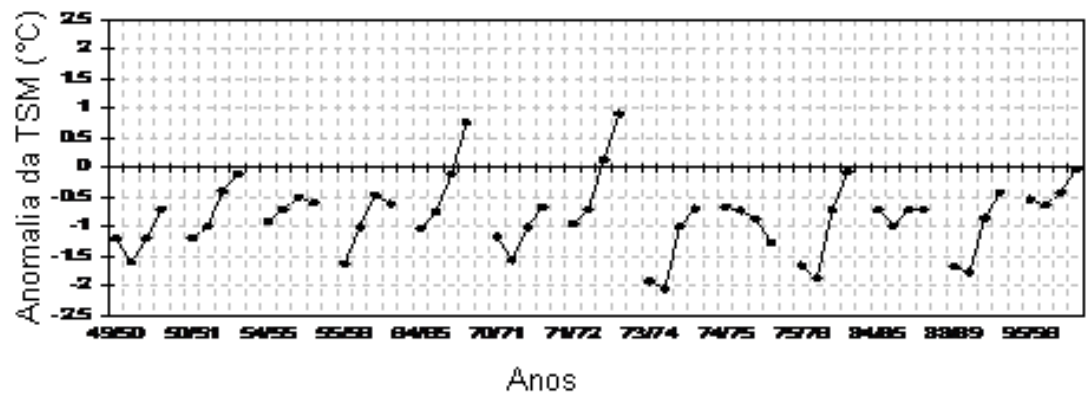

Figura 8 - Anomalia sazonal da TSM sobre a Região Niño 3.4 para anos de evento La Niña. Os trimestres são, respectivamente, SON, DJF, MAM e JJA para cada ano.

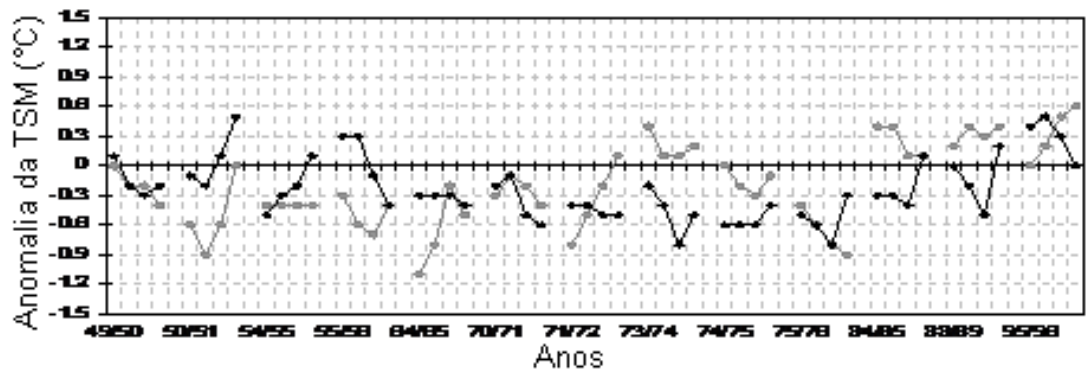

Figura 9 - Anomalia sazonal da TSM sobre o ATS (linha cinza) e o ATN (linha preta) para anos de evento La Niña. Os trimestres são, respectivamente, SON, DJF, MAM e JJA para cada ano. 
$73 / 74,74 / 75,84 / 85,88 / 89$, mas pode também ser observado que este gradiente está presente nos anos de 70/71 e 74/75.

A precipitação em anos de LN também apresentou uma grande variabilidade sobre as sub-regiões 1 e 2 (Figuras 10a e 10b). Nessas sub-regiões, após 1976, foram observadas precipitações acima da média com maiores anomalias que as do período anterior, a exceção ocorreu no ano de 1976 quando se observou, nas duas sub-regiões, redução das chuvas no período chuvoso. Verifica-se que, no período anterior a 1976, as anomalias de precipitação sobre a sub-região 1 oscila nos períodos chuvosos entre valores negativos e positivos, e sobre a sub-região 2 , as anomalias de precipitação também demonstram oscilações, porém essas são pequenas, podendo-se dizer que os períodos chuvosos ocorreram em torno da média. A subregião 3 (Figura 10c) apresentou, em anos de LN, anomalias de precipitação na maioria dos casos negativas ou em torno da média climatológica.

Diferente dos resultados encontrados em anos de EN na sub-região 1, a diferença AOGA-CPC nos eventos LN apresenta as menores diferenças (Figura 11). A exceção é para os anos

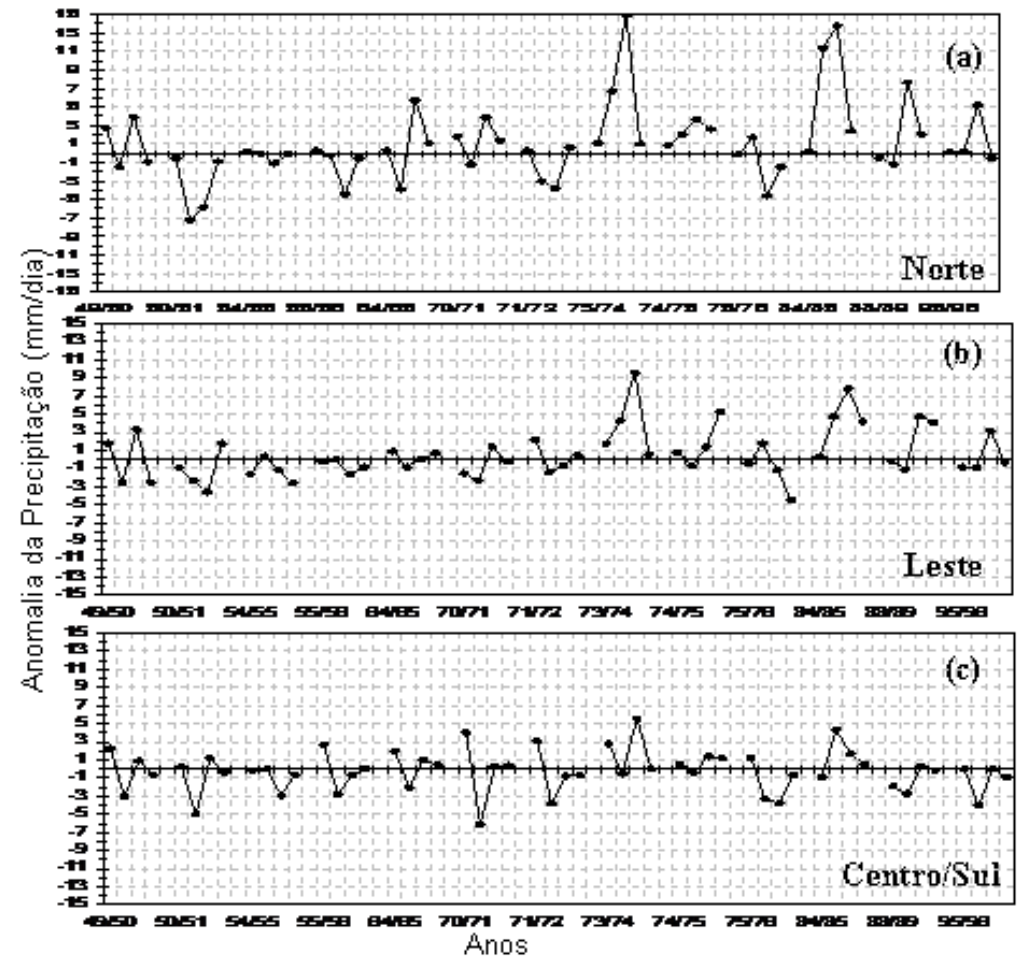

Figura 10 - Anomalia sazonal da Precipitação para as três sub-regiões sobre o NEB com os dados observados do CPC/NOAA para anos de evento La Niña. (a) Sub-região 1 - Norte; (b) Sub-região 2 - Leste e (c) Sub-região 3 - Centro/Sul. Os trimestres são, respectivamente, SON, DJF, MAM e JJA para cada ano.

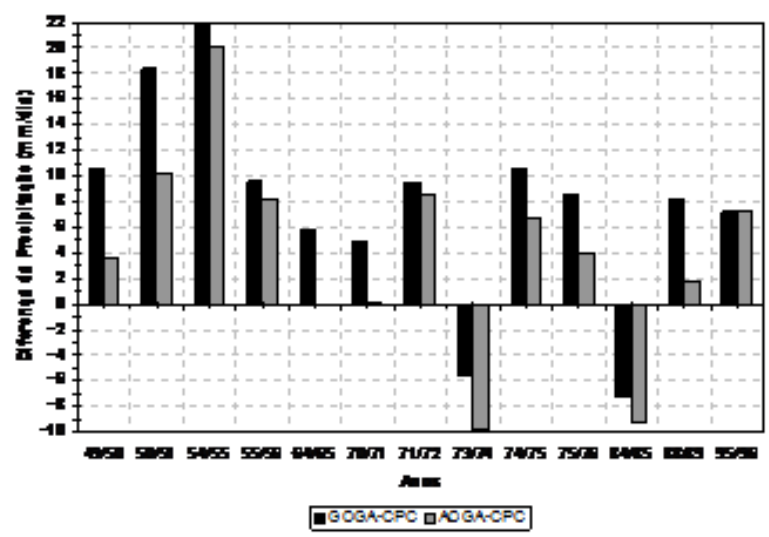

Figura 11 - Diferença da precipitação (FMAM) entre as simulações e os dados observados do CPC/NOAA (GOGA-CPC e AOGA-CPC), nos anos de La Niña para a sub-região 1. 
de 1974 e 1985 em que a diferença GOGA-CPC foi menor e para o ano de 1996 quando as diferenças foram idênticas. Assim, mesmo sabendo que essa sub-região sofre a influência das condições sobre o Pacífico, esses resultados mostram que as condições sobre o Atlântico contribuem para a precipitação nos anos de LN.

Analisando a Figura 12 para o período AMJJ sobre o leste do NEB (sub-região 2), observa-se que as diferenças oscilaram muito, mostrando assim, que essa sub-região necessita de estudos mais detalhados para verificar o impacto de cada evento e a ação conjunta dos oceanos Pacífico e Attântico sobre a precipitação. Da mesma forma que nos anos de eventos EN, essa sub-região apresenta os menores valores das diferenças entre a precipitação simulada e a observada.

A Figura 13 mostra altos valores da diferença entre GOGA-CPC e AOGA-CPC para a sub-região 3, resultado também verificado nos anos de EN. Entretanto, a figura revela que, em anos de LN, a diferença AOGA-CPC apresentou em 12 dos 13 casos os menores valores em relação à GOGA-CPC, sugerindo que nesses anos, essa sub-região é influenciada pelo Oceano Atlântico.

\subsection{Anos com Gradiente Meridional Positivo}

Nos anos com GRADM positivo, tal como esperado, observa-se que as anomalias sazonais da TSM sobre o ATN se apresentam, em geral, com valores positivos e negativos sobre o ATS (Figura 14), configurando assim, o gradiente da TSM apontando para o norte. É notado que as maiores diferenças entre as anomalias sobre o ATN e ATS são encontradas nos trimestres DJF e MAM, principalmente neste último. As anomalias da TSM sobre o Atlântico em comparação com as observadas no Pacífico durante o fenômeno ENOS são bem menores, atingindo um máximo de $\pm 0,9^{\circ} \mathrm{C}$.

Verifica-se na Figura 15 que, nos anos com GRADM positivo, a região do Niño 3.4 apresentou, na maioria dos casos, valores positivos associados ao evento EN configurado sobre o Pacífico.



Figura 12 - Diferença da precipitação (AMJJ) entre as simulações e os dados observados do CPC/NOAA (GOGA-CPC e AOGA-CPC), nos anos de La Niña para a sub-região 2 .

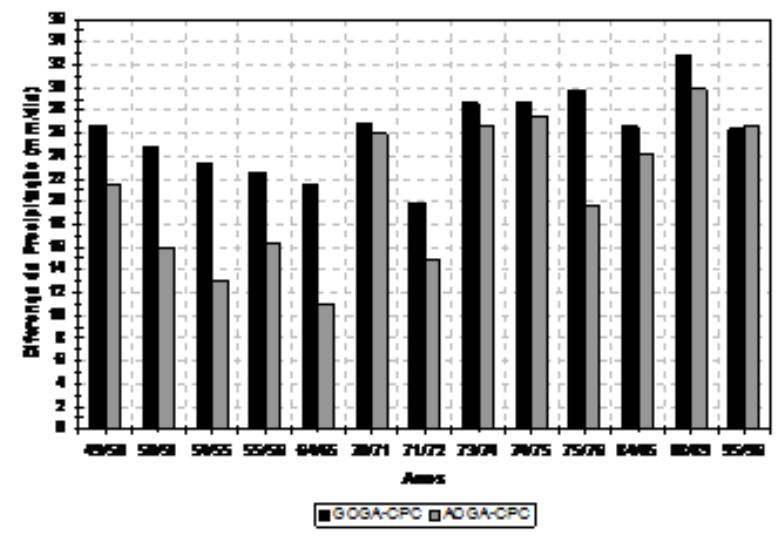

Figura 13 - Diferença da precipitação (NDJF) entre as simulações e os dados observados do CPC/NOAA (GOGA-CPC e AOGA-CPC) nos anos de La Niña para a sub-região 3. 


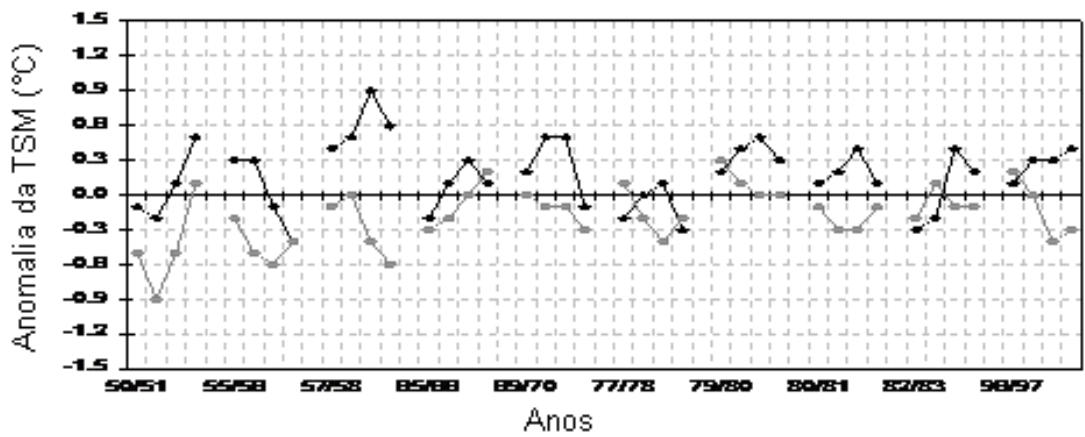

Figura 14 - Anomalia sazonal da TSM sobre o ATS (linha cinza) e o ATN (linha preta) para anos com GRADM positivo. Os trimestres são, respectivamente, SON, DJF, MAM e JJA para cada ano.

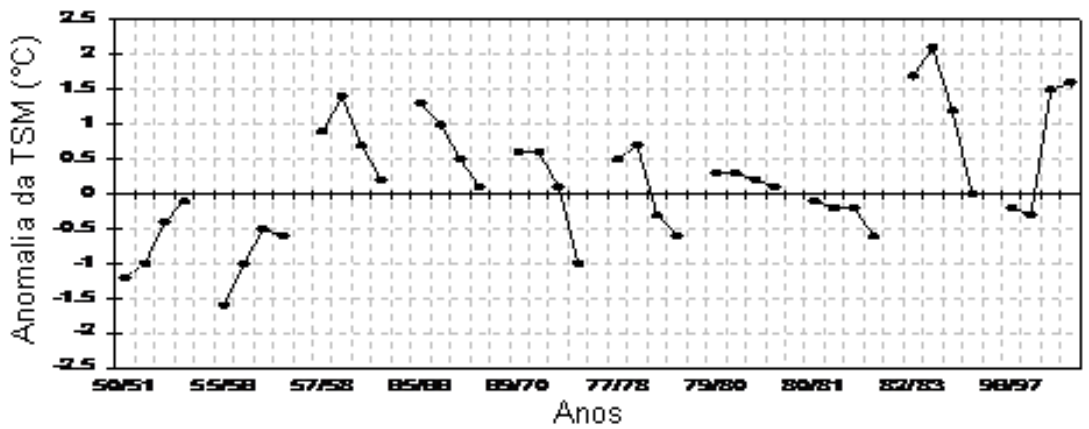

Figura 15 - Anomalia sazonal da TSM sobre a região Niño 3.4 para anos com GRADM positivo. Os trimestres são, respectivamente, SON, DJF, MAM e JJA para cada ano.

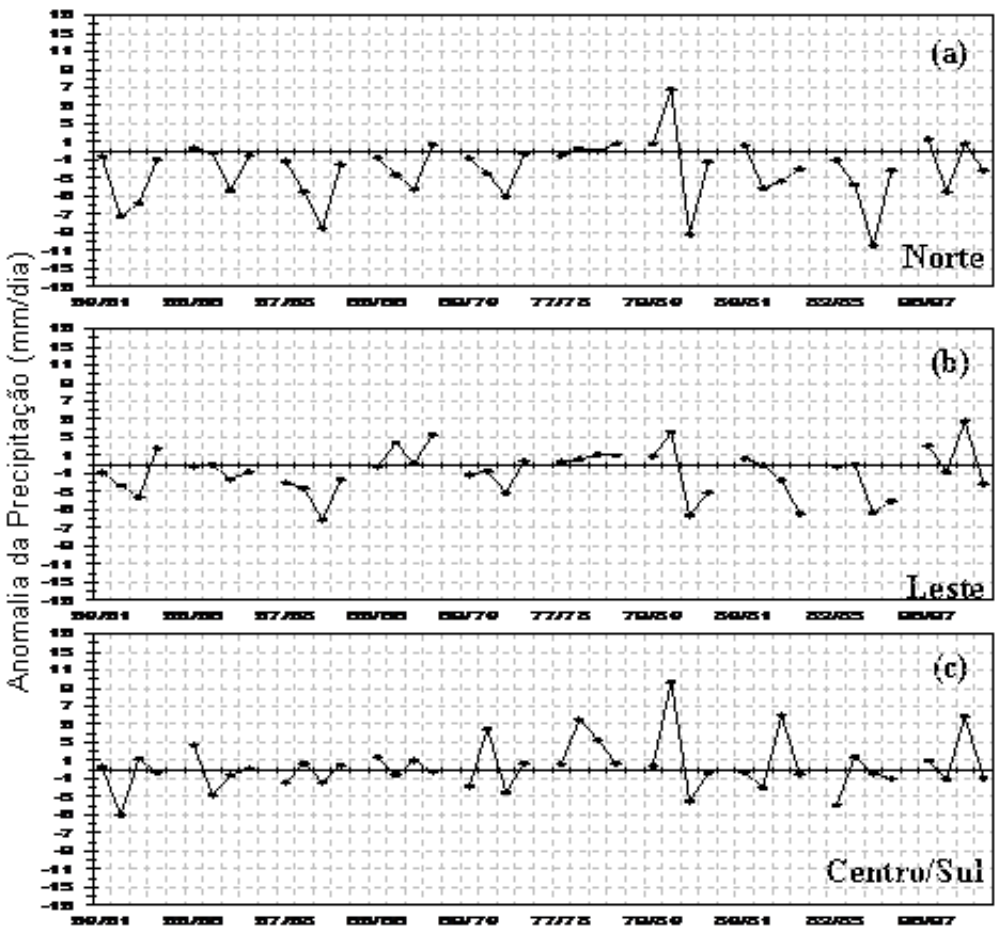

Figura 16 - Anomalia sazonal da precipitação para as três sub-regiões sobre o NEB com os dados observados do CPC/NOAA para 10 anos com GRADM positivo (gradiente para norte) sobre a bacia do Atlântico. (a) Sub-região 1 - Norte; (b) Sub-região 2 - Leste e (c) Sub-região 3 - Centro/ Sul. Os trimestres são, respectivamente, SON, DJF, MAM e JJA para cada ano. 
As anomalias sazonais da precipitação em anos de GRADM positivo apresentaram uma variabilidade bem menor em comparação aos anos de eventos ENOS, em que se observou uma alta variabilidade (vide Figuras 4 e 10). Nota-se que a estação chuvosa MAM para a sub-região 1 (Figura 16a) é afetada com anomalias negativas de precipitação em praticamente todos os anos, com exceção apenas dos anos de 1978 e 1997, quando foram observados valores em torno da média climatológica. Esse resultado está coerente com estudos anteriores (Nobre e Shukla, 1996; dentre vários outros), os quais mostram que este GRADM provoca o deslocamento da ZCIT para sua posição mais ao norte e, em conseqüência, existe uma redução da precipitação na estação chuvosa do norte do NEB. Além desse fator, quando o GRADM era positivo, também foi observado o predomínio de eventos EN que, juntamente com este gradiente, atua no sentido de reduzir as chuvas sobre a estação chuvosa da sub-região. A sub-região 2 (leste) também sofre o impacto desse GRADM positivo, podendo ser observado na Figura 16b, que a precipitação abaixo da normal é uma característica, porém em alguns anos, como 1965/66, além de 1950/51 e
1996/97, apresenta valores positivos ou oscilando entre positivo e negativo. Observa-se que a sub-região 3 (Figura 16c) parece não ser influenciada apenas pelo GRADM positivo, notando-se pequenas anomalias oscilando entre valores negativos e em torno da média, no período antes de 1970 e após 1980. Na década de 70 observa-se que as anomalias de precipitação são positivas apresentando valores maiores do que o resto do período.

A diferença da precipitação simulada pelos dois experimentos do modelo ARPEGE no período chuvoso de cada sub-região com a precipitação observada é mostrada nas Figuras 17, 18 e 19 (respectivamente para a sub-região 1, 2 e 3). Observa-se que, para a sub-região 1 (Figura 17), a diferença GOGA-CPC apresentou-se com menores valores para 4 anos, e a diferença AOGA-CPC para o mesmo número de anos, e, em dois casos, elas apresentaram a mesma diferença. Esses resultados confirmam estudos anteriores, os quais mostram que a sub-região 1 sofre o impacto do deslocamento da ZCIT em conseqüência do GRADM positivo, bem como do fenômeno EN que predominou nesses anos.

Para a sub-região leste (Figura 18), são observados os menores valores de diferenças entre GOGA-CPC e AOGA-CPC,

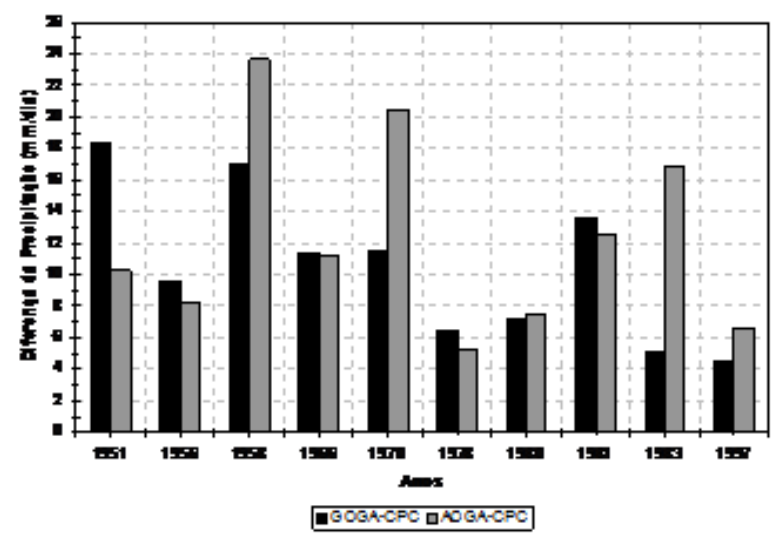

Figura 17 - Diferença da precipitação (FMAM) entre as simulações e os dados observados do CPC (GOGA-CPC e AOGA-CPC), nos anos com GRADM positivo para a sub-região 1 .

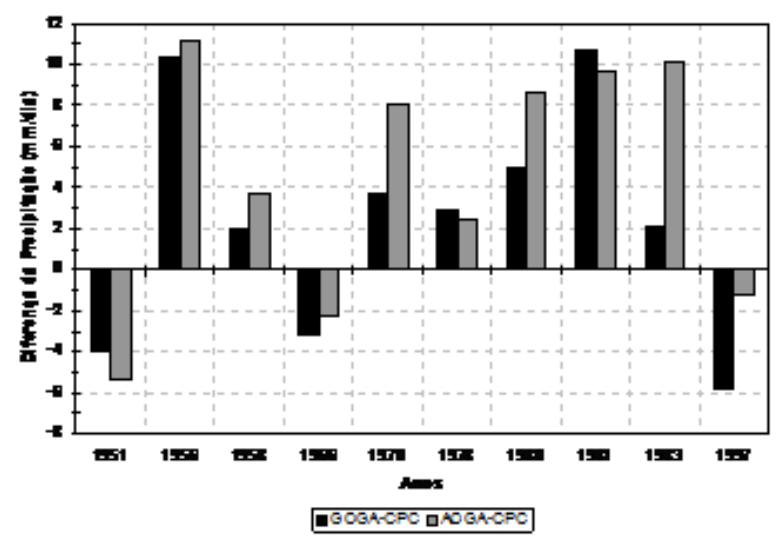

Figura 18 - Diferença da precipitação (AMJJ) entre as simulações e os dados observados do CPC (GOGA-CPC e AOGA-CPC), nos anos com GRADM positivo para a sub-região 2 . 
como vem sendo encontrado nos resultados anteriores para anos de ENOS. Observa-se que as diferenças entre GOGA-CPC e AOGA-CPC oscilam bastante.

A sub-região 3 continua a mostrar os altos valores da diferença da precipitação GOGA-CPC e AOGA-CPC (Figura 19), e, embora essas diferenças encontrem-se bem próximas, as exceções são visualizadas nos anos de 1951, 1956 e 1978, quando as diferenças são um pouco maiores e AOGA-CPC apresentou os menores valores.

\subsection{Anos com Gradiente Meridional Negativo}

Nesses anos com GRADM negativo, observam-se valores de anomalias da TSM sazonais positivas sobre o ATS e negativas no ATN, configurando este gradiente com sentido para sul (Figura 20). Da mesma forma que encontrado para o GRADM positivo, os maiores valores entre as duas bacias sobre o Atlântico são encontrados nos meses de MAM. Nesses eventos, predominaram sobre o oceano Pacífico anos de LN (Figura 21).



Figura 19 - Diferença da precipitação (NDJF) entre as simulações e os dados observados do CPC (GOGA-CPC e AOGA-CPC), nos anos com GRADM positivo para a sub-região 3 .

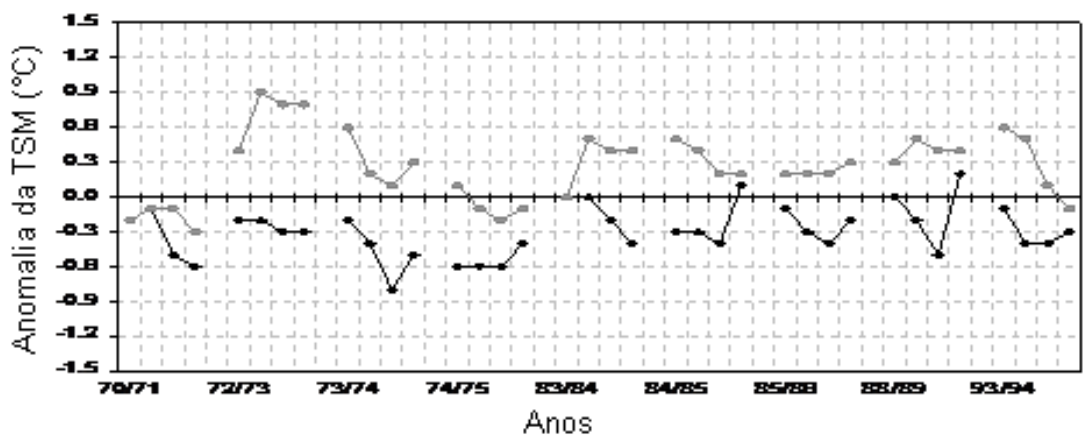

Figura 20 - Anomalia sazonal da TSM sobre o ATS (linha cinza) e o ATN (linha preta) para anos com GRADM negativo. Os trimestres são, respectivamente, SON, DJF, MAM e JJA para cada ano.

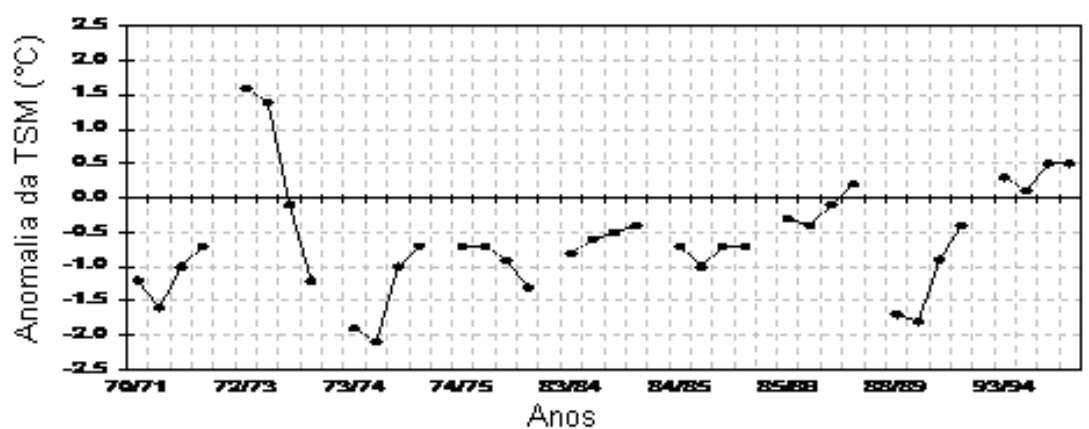

Figura 21 - Anomalia sazonal da TSM sobre a Região Niño 3.4 para anos com GRADM negativo. Os trimestres são, respectivamente, SON, DJF, MAM e JJA para cada ano. 
As anomalias sazonais de precipitação para a subregião 1 e 2 (Figura 22a e 22b) mostram a grande influência do gradiente meridional negativo sobre a precipitação, isso é explicado pela localização da ZCIT na sua posição mais ao sul, favorecendo, assim, a precipitação sobre o NEB, principalmente sobre essas sub-regiões. Vale ressaltar que não só o oceano Atlântico apresenta condições favoráveis à precipitação. Como dito anteriormente, observa-se, na maioria dos anos, sobre o Pacífico a configuração da LN que, em geral, também influencia positivamente na precipitação do NEB. Sobre a sub-região 3, verificam-se anomalias oscilando em torno da média, com maiores anomalias negativas observadas nos anos de 1970/71 e 1983/84 e anomalias positivas nos anos de 1984/85.

As diferenças entre as precipitações simuladas e as observadas são visualizadas nas Figuras 23, 24 e 25, respectivamente para as sub-regiões 1,2 e 3 . Nota-se para a sub-região 1 (norte), que nos anos com GRADM negativo são obtidos os menores valores das diferenças em comparação aos outros casos de eventos ENOS e GRADM positivo. Contudo, os resultados mostram que há uma oscilação nos menores valores

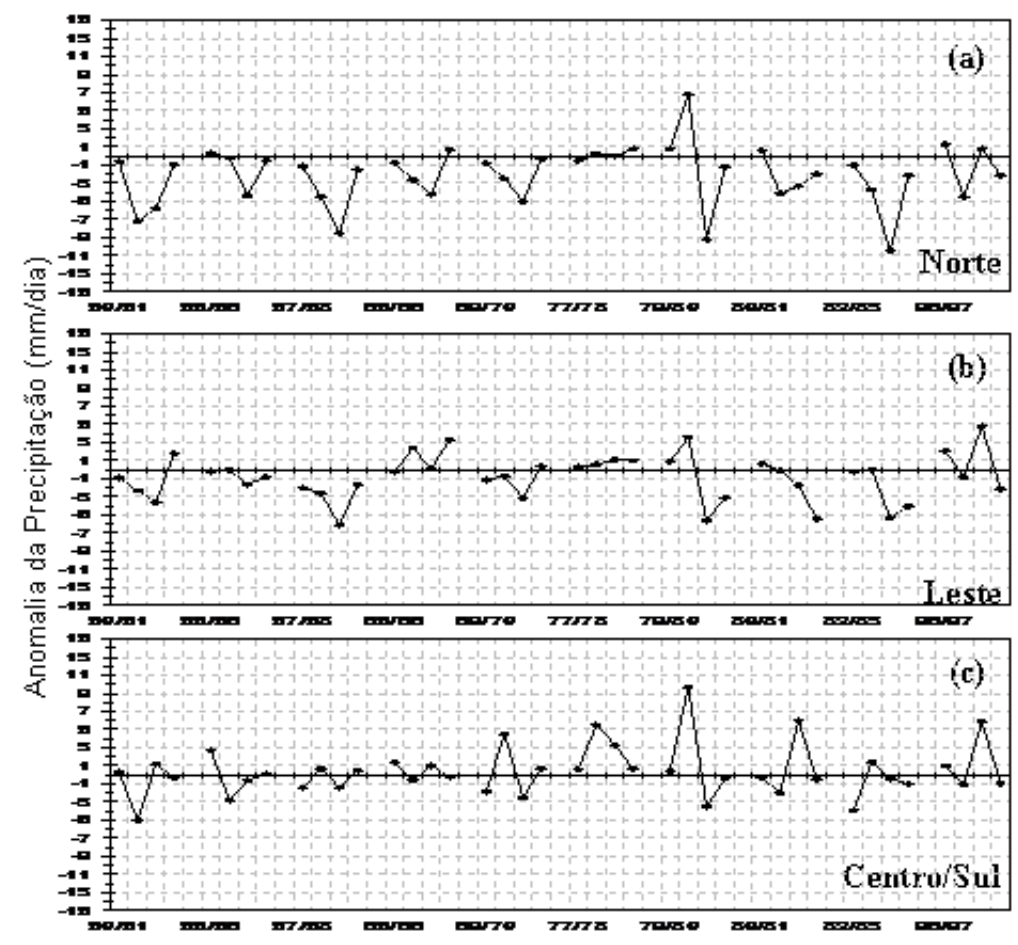

Figura 22 - Anomalia sazonal da precipitação para as três sub-regiões sobre o NEB com os dados observados do CPC/NOAA para 09 anos com GRADM negativo sobre a bacia do Atlântico. (a) Sub-região 1 - Norte; (b) Sub-região 2 - Leste e (c) Sub-região 3 - Centro/Sul. Os trimestres são, respectivamente, SON, DJF, MAM e JJA para cada ano.

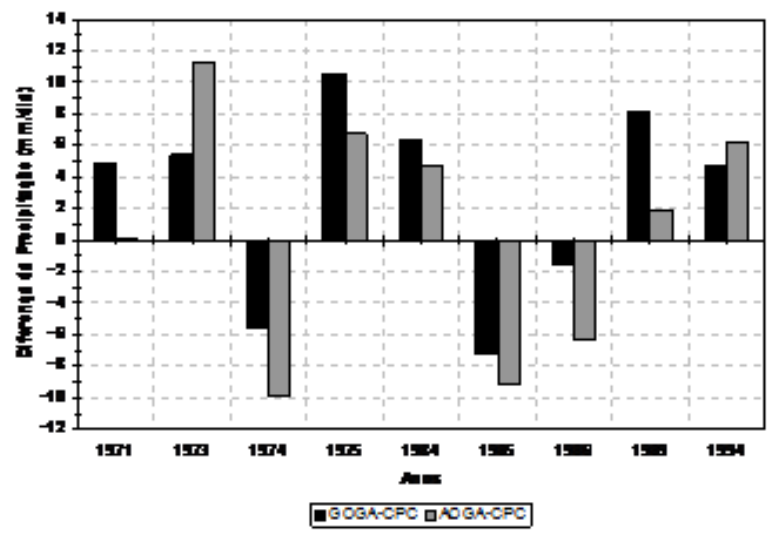

Figura 23 - Diferença da precipitação (FMAM) entre as simulações e os dados observados do CPC (GOGA-CPC e AOGA-CPC), nos anos com GRADM negativo para a sub-região 1 . 
apresentados pela diferença entre as simulações e a precipitação observada.

Na sub-região 2 (Figura 24), foi observado, em todos os eventos, que ela apresenta os menores valores das diferenças da precipitação. Semelhante ao encontrado para a sub-região 1 , as diferenças oscilam dependendo dos anos. Observa-se, em conformidade com os eventos discutidos anteriormente, que no centro/sul (sub-região 3), são encontradas as maiores diferenças entre as precipitações simuladas e observadas. Nessa sub-região (Figura 25) nota-se que as diferenças entre as duas simulações e as observadas são bem próximas, demonstrando que o Atlântico apresenta as maiores influências, já que a simulação AOGA que suprime os eventos extremos sobre o Pacífico é bem próxima da GOGA que inclui esses eventos (EN e LN).

\section{CONCLUSÕES}

Em resumo, tem-se que em anos de EN e LN a precipitação sobre as três sub-regiões apresenta alta variabilidade, o que não foi observado nos anos com GRADM positivo ou negativo, que demonstra um resultado praticamente "direto". O impacto sobre a precipitação observada na sub-região 1 (norte do NEB), em anos de EN, é bem caracterizado por anomalias negativas (período de secas) como descritas na literatura (Molion e Bernardo, 2002; Pezzi e Cavalcanti, 2001). Em anos de LN, observa-se, na sub-região 1 e 2, dois períodos com impactos contrastantes, antes de 1976, com predomínio de anomalias de precipitação negativas (redução das chuvas), e após este ano, anomalias positivas (chuvas acima da média climatológica), além de apresentarem valores maiores do que o período anterior, que podem estar relacionados às mudanças ocorridas nesta época (segunda metade do século 20) tais como, mudança na fase da Oscilação Decadal do Pacífico (Graham, 1994; Stenseth et al., 2003) e mudança na fase da Oscilação do Atlântico Norte (Hurrell e van Loon, 1997; Stenseth et al., 2003; Wainer et al., 2008). A sub-região 3 mostra impactos contrários aos encontrados em geral para as outras sub-regiões, ou seja, em anos de EN anomalias variando da normal a acima, e nos anos de LN anomalias oscilando da normal a abaixo. As sub-regiões 1 e 2 em anos com GRADM negativo apresentam anomalias

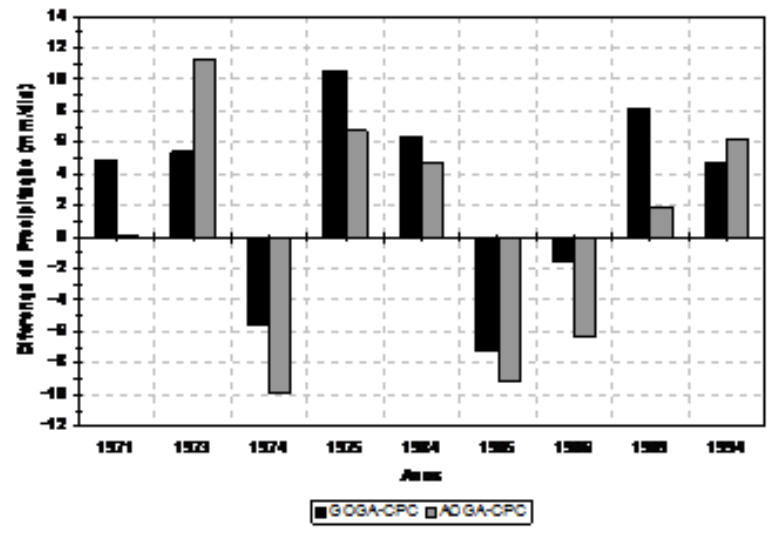

Figura 24 - Diferença da precipitação (AMJJ) entre as simulações e os dados observados do CPC (GOGA-CPC e AOGA-CPC), nos anos com GRADM negativo para a sub-região 2 .



Figura 25 - Diferença da precipitação (NDJF) entre as simulações e os dados observados do CPC (GOGA-CPC e AOGA-CPC), nos anos com GRADM negativo para a sub-região 3 . 
de precipitação positivas, situação contrária observou-se nos anos de GRADM positivo, ou seja, anomalias de precipitação negativas.

Com relação à diferença da precipitação entre as simulações do modelo ARPEGE e os dados observados do $\mathrm{CPC}$, observa-se, em geral, que, sobre as sub-regiões 1 e 2 em anos de EN a diferença GOGA-CPC apresenta os menores valores, ou seja, tem-se a ação conjunta dos oceanos globais. Nos anos de LN, observa-se o contrário, a diferença AOGA-CPC mostra os menores valores, mostrando assim a contribuição do Atlântico. Em todos os eventos são verificados os menores valores da diferença entre GOGA-CPC e AOGA-CPC sobre a sub-região 2 (leste do NEB). Os resultados mais críticos são para a sub-região 3 (centro/sul do NEB), onde se observa as maiores diferenças. É bom recordar que essa sub-região possui vários regimes de precipitação diferentes (como por exemplo, o semi-árido nordestino e uma parte do sudeste), dessa forma seria interessante uma melhor divisão para uma análise mais detalhada.

Todos os resultados obtidos mostram que os eventos extremos, tanto no Pacífico quanto no Attântico, influenciam a precipitação sobre as sub-regiões do NEB, mas não de maneira igual e direta. Dessa forma, são necessários estudos mais detalhados, observando-se as condições oceânicas e atmosféricas em anos chuvosos e secos com o intuito de tentar compreender as reais contribuições de cada evento sobre cada uma das áreas estudadas.

\section{AGRADECIMENTOS}

Os autores agradecem aos revisores da RBMET pelos comentários e sugestões que foram de grande importância para o aprimoramento deste trabalho. Agradecem também ao Dr. Christophe Cassou (CNRS-CERFACS) pela concessão dos dados do modelo ARPEGE. Esse trabalho faz parte do Projeto de cooperação cientifico franco-brasileiro IRD-CNPq CATIN "Climat de l'Atlantique Tropical et Impacts sur le Nordeste" (Processo CNPq 492690/2004-9), como também do Projeto Institucional da UFCG CAMISA "Clima do Nordeste e do Atlântico Tropical e o Monitoramento dos Impactos no Semi-Árido". Esse trabalho faz parte dos resultados da tese de doutorado do primeiro autor.

\section{REFERÊNCIAS BIBLIOGRÁFICAS}

ACEITUNO, P. On the functioning of the Southern Oscillation in the South American Sector. Part I: Surface Climate. Monthly Weather Review, v. 116, n.3, p. 505-524, 1988. ANDREOLI, R.V. Variabilidade e Previsibilidade da Temperatura da Superfície do Mar no Atlântico
Tropical. 224 p. (INPE-9557-TDI/833). Tese (Doutorado em Meteorologia)-Instituto Nacional de Pesquisas Espaciais, São José dos Campos, 2002.

ANDREOLI, R.V.; KAYANO, M.T. Multi-scale variability of sea surface temperature in the tropical Atlantic. Journal of Geophysical Research, 109, 2004.

ARAGÃO, J.O.R. A influência dos Oceanos Pacífico e Atlântico na Dinâmica do Tempo e do Clima do Nordeste do Brasil. Capítulo do Livro Oceanografia um cenário tropical. Org.: Eskinazi-Leça, E.; Neumann-Leitão; Costa, M.F.da. Universidade Federal de Pernambuco. Recife, 2004.

CASSOU, C. Rôle de l'Océan dans la Variabilité Basse Fréquence de l'Atmosphère sur la Région Nord Atlantique-Europe. Thèse de doctorat. Toulouse: France. 280 p. 2001.

CHEN, M.et al. Global Land Precipitation: A 50-yr Monthly Analysis Based on Gauge Observations, Journal of Hydrometeorology, 3: 249-266, 2002.

DÉQUÉ, M. et al. The climate version of the ARPEGE/IFS: a contribution to the French community climate modelling, Climate Dynamics, 10: 249-266, 1994.

GIANNINI, A.; CANE, M.A.; KUSHNIR,Y. Interdecadal changes in the ENSO teleconnection to the Caribbean region and the north Atlantic oscillation. Journal Climate, 14, 2867-2879, 2001.

GIANNINI, A.; SARAVANAN, R.; CHANG, P. The predictioning role of Tropical Atlantic variability in the development of the ENSO teleconnection: implications for the prediction of Nordeste rainfall. Climate Dynamics, 22(8): 839-855, 2004.

GRAHAM, N. E. Decadal-scale climate variability in the tropical and North Pacific during the 1970s and 1980s: Observations and model results. Climate Dynamics, vol. 10: 135-162, 1994.

HANLEY, D.E.et al. A quantitative evaluation of ENSO indices. Journal of Climate, v.16, n.8, p.1249-1258, 2003.

HASTENRATH, S. Variations in low-latitude circulation and extreme climatic events in the tropical Americas. Journal of Atmospheric Sciences, 33(2): 202-215, 1976.

HASTENRATH, S. On modes of tropical circulation and climate anomalies. Journal of Atmospheric Sciences, 35(12): 2222-2231, 1978.

HASTENRATH, S.; HELLER, L. Dynamics of climatic hazards in Northeast Brazil. Quarterly Journal of the Royal Meteorological Society, vol. 102, 77-92, 1977.

HURRELL, J. W.; van LOON, H. Decadal variations in climate associated with the North Atantic Oscillation. Climatic Change, vol. 36, pp. 301-326, 1997.

KAYANO, M.T.; ANDREOLI, R.V. Decadal variability of northern northeast Brazil rainfall and its relation to tropical 
sea surface temperature and global sea level pressure anomalies. Journal Geophysical Research-Oceans, v. 109, n. C11011, Nov. 2004.

KAYANO,M.T.; RAO, V.B.; MOURA,A.D. Tropical circulations and the associated rainfall anomalies during two contrasting years. Journal of Climatology, 8(5) 477-488, 1988.

KILADIS, G. N.; DIAZ, H. D. Global climatic anomalies associated with extremes in the Southern Oscillation. Journal of Climate, 2(9): 1069-1090, 1989.

KOUSKY, V.E.; GAN, M.A. Upper tropospheric cyclonic vortices in the tropical South Atlantic. Tellus, 6(33), 538-551, 1981.

KOUSKY, V.E.; KAYANO, M. T. Principal modes of outgoing longwave radiation and 250-mb circulation for the South American sector. Journal of Climate, 7, 1131-1143, 1994.

KOUSKY, V.E.; KAYANO, M.T.; CAVALCANTI, I.F.A. A review of the Southern Oscillation: oceanic-atmospheric circulation changes and related rainfall anomalies. Tellus, 36A: 490-504, 1984.

KOUSKY, V.E.; ROPELEWSKI, C.F. Extremes in the Southern oscillation and their relationship to precipitation anomalies with emphasis on the South American region. Revista Brasileira de Meteorologia, 4(2): 351-362, 1989.

MARKHAM, C.G.; MAcLAIN, P.R. Sea surface temperature related to rain in Ceará, Northeast Brazil. Nature, 265 (5592): 320-322, 1977.

MARQUES, V. S.; RAO, V. B; MOLION, L. C. B. Interannual and seasonal variation in the structure and energetics of the atmosphere over Northeast Brazil. Tellus, v. 35A, n. 2, p. 136-148, Mar. 1983.

MÉLICE, J.L.; SERVAIN, J. The Tropical Atlantic Meridional SST Gradient Index and its relationships with SOI, NAO and Southern Ocean. Climate Dynamics, v. 20, n.5, p. 447-464, 2003.

MOLION, L.C.B.; BERNARDO, S.O. Uma revisão da dinâmica das chuvas no Nordeste Brasileiro. Revista Brasileira Meteorologia, 17(1)1-10, 2002.

MOURA, A.D.; SHUKLA, J. On the dynamics of droughts in northeast Brazil: observations, theory e numerical experiments with a general circulation model. J. Atmos. Sci., 38, 2653 - 2675, 1981.

NOBRE, C.A.; MOLION, L.C.B. The climatology of drought prediction. Kluwer Academic Publishers. The impact of variation on agriculture, v. 2, 1988.

NOBRE, P.; SHUKLA, J. Variation of sea surface temperature, wind stress and rainfall over the tropical Atlantic and South America. Journal of Climate, 9, 2464-2479, 1996.

PENLAND, C.; MATROSOVA, L. Prediction of tropical Atlantic sea surface temperature using linear inverse modeling. Journal of Climate, v. 11, p. 483-496, 1998.
PEZZI, L.P.; CAVALCANTI, I.F.A. The relative importance of ENSO and tropical Atlantic sea surface temperature anomalies for seasonal precipitation over South America: A numerical study. Climate Dynamics, 17: 205-212, 2001. RASMUSSON, E.M.; CARPENTER, T.H. Variations in Tropical sea surface temperature and surface wind fields associated with the Southern Oscillation/El Niño. Monthly Weather Review, 110: 354-384, 1982.

RAO, V.B.; HADA, K. Characteristics of rainfall over Brazil: annual variations and connections with the southern oscillation. Theoretical and Applied Climatology, 42(1): 81-91, 1990.

ROPELEWSKI, C.F.; HALPERT, M.S. Global and regional scale precipitation patterns associated with El Niño/ Southern Oscillation. Monthly Weather Review, 115: 2352-2362, 1987.

ROPELEWSKI, C.H.; HALPERT, M.S. Precipitation patterns associated with the high index phase of Southern Oscillation. Journal of Climate, 2 (3): 268-284, 1989.

SARAVANAN, R.; CHANG, P. Interaction between tropical Atlantic variability and El Nino-Southern Oscillation. Journal of Climate.v.13, n. 13, p. 2177 - 2194, 2000.

SERVAIN, J. Simple climatic indices for the tropical Atlantic Ocean and some applications. J. Geophys. Res., 96, 15.137 - 15.146, 1991.

STENSETH, N. C.et al. Studying climate effects on ecology through the use of climate indices: the North Atlantic Oscillation, El Niño Southern Oscillation and beyond. Proceeding Royal Society London, Published online, doi: 10.1098/rspb.2003.2415., 10 pp, 2003.

SUTTON, R.T.; JEWSON, S.P.; ROWELL, D.P. The elements of climate variability in the tropical Atlantic region. Journal of Climate, 13: 3261-3284. 2000.

TRENBERTH, K.E. The definition of El Niño. Bulletin American Meteorological Society, 78: 2771-2777, 1997.

UVO, C. A Zona de Convergência e sua relação com a precipitação no Nordeste Brasileiro. São José dos Campos. 120 p. (INPE-4887-TDL/378). Dissertação de Mestrado. Instituto Nacional de Pesquisas Espaciais-INPE. 1989.

WAINER, I.; SERVAIN, J.; CLAUZET, G. Is the decadal variability in the tropical Atlantic a precursor to the NAO? Annales Geophysicae. vol. 26, pp. 4075-4080, 2008.

WANG, C. Atmospheric Circulation cells associated with the El Niño-Southern Oscillation. Journal of Climate, 15: 399-419, 2002a.

YAMAZAKI, Y.; RAO, V.B. Tropical cloudiness over the South Atlantic Ocean. Journal Meteorological Society of Japan, 55, 205-207, 1977. 